Q. DING

KODAI MATH. J.

17 (1994), 320-328

\title{
ON SPECTRAL CHARACTERIZATIONS OF MINIMAL HYPERSURFACES IN A SPHERE
}

\author{
BY QING DING
}

\begin{abstract}
Let $M$ be a closed minimal hypersurface in an Euclidean sphere $S^{n+1}(1)$. We first prove that a minimal isoparametric hypersurface $M$ in a 4-dimensional sphere is completely determined by its spectrum $\operatorname{Spec}^{p}(M)$, here $p \in\{0,1,2,3\}$. In higher dimensional sphere, we prove that if $\operatorname{Spec}^{p}(M)=\operatorname{Spec}^{p}\left(M_{m, n-m}\right)$ for $p=0,1$, where

$$
M_{m, n-m}=S^{m}\left(\sqrt{\frac{m}{n}}\right) \times S^{n-m}\left(\sqrt{\frac{n-m}{n}}\right)
$$

is a Clifford torus, then $M$ is $M_{m, n-m}$. Furthermore, we prove that $M_{n, n} \rightarrow$ $S^{2 n+1}(1)(n \geqq 4)$ is also characterized by $\operatorname{Spec}^{p}\left(M_{n, n}\right)$ for some $p=p(n)$.
\end{abstract}

\section{§ 1. Introduction}

For a smooth compact, oriented Riemannian manifold $M$ of dimension $n$, let $\Lambda^{p}(M)$ denote the space of $C^{\infty}$ differential forms of degree $p=0,1, \cdots, n$ with real coefficients. The Laplace operator $\Delta$ of $M$ acting on functions has a natural generalization to $\Lambda^{p}(M)$. In the theory of spectrum of Laplace operator on $\Lambda^{p}(M)$, one can see that the interplay among analysis, topology and geometry is even striking (e.g., see [6]). We denote by $\operatorname{Spec}^{p}(M)$ the spectrum of Laplace operator on $\Lambda^{p}(M)$.

It is interesting to see the relation of $\operatorname{Spec}^{p}(M)$ and the geometry on $M$, which gives rise to the following old question: Does $\operatorname{Spec}^{p}(M)$ determine the geometry of Riemannian manifold $M$ ? The answer to this problem in general case is negative. This is a consequence of the counter example which is given by Milnor in [10]. So the problem is divided into two directions. One direction is to find new counter examples. A series studies along this line have been done by Vigneras [13], Ikeda [8] and others. Another direction is to give an affirmative answer for a special Riemannian manifold. The studies of this direction have also been done by Berger [1], Patodi [11], Tanno [12] and many others.

Received November 8, 1993 ; revised January 5, 1994. 
In this paper, we will deal with the latter problem on minimal hypersurfaces in an Euclidean sphere. In his paper [5], Donnelly gave a spectral characterization of the totally geodesic minimal submanifold in a sphere. A further study of this aspect was done by Hasegawa, he characterized some concrete minimal submanifolds in a sphere by the spectrum, particularly Veronese manifolds. And he also characterized Clifford tori by their spectrum with some additional geometric conditions (see [7] for details). On the other hand, from the recent work of Chang [2] (or of Cheng and Wan [3]), we know that the totally geodesic 3-sphere, Clifford torus and Cartan's minimal hypersurface are the only closed minimal hypersurfaces of 4 -sphere $S^{4}(1)$ with constant scalar curvature. For these minimal isoparametric hypersurfaces in $S^{4}(1)$, we can give a spectral characterization as follows.

THEOREM 1. Let $M$ be a closed minimal hypersurface in $S^{4}(1)$. If $\operatorname{Spec}^{p}(M)$ $=\operatorname{Spec}^{p}\left(M_{0}\right)$ for a given $p(0 \leqq p \leqq 3)$, where $M_{0}$ is the totally geodesic 3-space, or Clifford torus $S^{1}(\sqrt{1 / 3}) \times S^{2}(\sqrt{2 / 3})$, or Cartan's minimal hypersurface. Then $M$ is nothing but $M_{0}$.

We also know that the Clifford tori $M_{m, n-m}=S^{m}(\sqrt{m / n}) \times S^{n-m}(\sqrt{n-m / n})$ $(1 \leqq m \leqq n-1)$ are the only closed minimal hypersurfaces of $S^{n+1}(1)$ with the scalar curvature $=n(n-1)-n$ (see [4]). For these minimal hypersurfaces, we like to give a spectral characterization without any additional geometric conditions. Namely, we have

THEOREM 2. Let $M$ be a closed minimal hypersurface in $S^{n+1}(1)$. If $\operatorname{Spec}^{p}(M)=\operatorname{Spec}^{p}\left(M_{m, n-m}\right)$ for $p=0$ and 1 , then $M$ is $M_{m, n-m}$.

Among the all Clifford tori, we will pay a special attention to $S^{n}(\sqrt{1 / 2}) \times$ $S^{n}(\sqrt{1 / 2})=M_{n, n}$ in $S^{2 n+1}(1)$. Berger et al. [1] proved that $S^{1} \times S^{1}$ is completely determined by $\operatorname{Spec}^{0}\left(S^{1} \times S^{1}\right)$ or $\operatorname{Spec}^{1}\left(S^{1} \times S^{1}\right)$. Hasegawa [7] proved that if $M$ is a minimal hypersurface in $S^{5}(1)$ satisfying $\operatorname{Spec}^{0}(M)=\operatorname{Spec}^{0}\left(M_{2,2}\right)$ and its Euler number $\chi(M) \leqq 4=\chi\left(M_{2,2}\right)$, then $M=M_{2,2}$. Tanno and Masuda [12] proved that if $\operatorname{Spec}^{0}(M \times N)=\operatorname{Spec}^{0}\left(S^{3} \times S^{3}\right)$, then $M($ or $N)$ is isometric to $S^{3}$. For $n \geqq 4$, we obtain the following.

THEOREM 3. Let $M$ be a closed minımal hypersurface in $S^{2 n+1}(1)(n \geqq 4)$ with $\operatorname{Spec}^{p}(M)=\operatorname{Spec}^{p}\left(M_{n, n}\right)$ for some $p=p(n)$ (e.g., $p$ is chosen in (3.23), (3.24) below). Then $M$ is $M_{n, n}$.

We will first set up notations and present some formulas and basic results of minimal hypersurfaces in a sphere in $\S 2$, and the proofs of the above theorems will be given in $\S 3$. 
QING DING

\section{§2. Preliminaries}

Throughout this paper unless otherwise stated, let $M$ be an $n$ dimensional hypersurface in an Euclidean sphere $S^{n+1}(1)$ to have no boundary and to be compact, connected, and of class $C^{\infty}$. Let $R, \hat{R}$ and $\rho$ be respectively the Riemann curvature tensor, Ricci curvature tensor and scalar curvature of $M$. We denote by $R_{\imath j k l}$ (or a similar way to $\hat{R}$ ) the components of $R$. The Gauss equation asserts that:

$$
R_{\imath j k l}=\delta_{i k} \delta_{j l}-\delta_{i l} \delta_{j k}+h_{i k} h_{j l}-h_{i l} h_{j k}
$$

where $\delta_{i}$, is the Kronecker symbol and $\left(h_{\imath \jmath}\right)$ the components of the second fundamental form of $M$ in $S^{n+1}(1)$.

For any fixed point $x_{0} \in M$, we can choose a frame field $e_{1}, \cdots, e_{n}$ such that $\left(h_{\imath \jmath}\right)$ is diagonalized at that point, say

$$
h_{\imath j}=\lambda_{i} \delta_{i j} .
$$

Let $h=\sum_{\imath=1}^{n} h_{i i}=\sum_{\imath=1}^{n} \lambda_{\imath}$ be the mean curvature of $M$ and $S=\sum_{\imath, j}^{n} h_{\imath j}^{2}=\sum_{\imath=1}^{n} \lambda_{\imath}^{2}$ the square of the length of the second fundamental form. Then we have

$$
\begin{gathered}
R_{\imath j k l}=\left(1+\lambda_{\imath} \lambda_{j}\right)\left(\delta_{i k} \delta_{j l}-\delta_{i l} \delta_{j k}\right), \\
\hat{R}_{\imath j}=\left[(n-1)+h \lambda_{i}-\lambda_{\imath} \lambda_{\jmath}\right] \delta_{i \jmath}, \\
\rho=n(n-1)+h^{2}-S .
\end{gathered}
$$

Therefore, the squares of the length of $R$ and $\tilde{R}$ are

$$
\begin{aligned}
& |R|^{2}=2 S^{2}-2 \sum_{i=1}^{n} \lambda_{i}^{4}+4 h^{2}-4 S+2 n(n-1), \\
& |\widetilde{R}|^{2}=h^{2} S+\sum_{i=1}^{n} \lambda_{i}^{4}+n(n-1)^{2}-2 h \sum_{i=1}^{n} \lambda_{i}^{3}+2(n-1) h^{2}-2(n-1) S
\end{aligned}
$$

where $\sum_{\imath=1}^{n} \lambda_{\imath}^{3}$ and $\sum_{\imath=1}^{n} \lambda_{\imath}^{4}$ are globally defined functions on $M$.

Since $M$ is compact, for $p=0,1, \cdots, n$, we set

$$
\operatorname{Spec}^{p}(M)=\left\{0 \leqq \lambda_{0, p} \leqq \lambda_{1, p} \leqq \cdots \uparrow+\infty\right\} .
$$

For those discrete eigenvalues, we have the Minakshisundaram-Pleijel's asymptotic expansion formula as follows:

$$
\sum_{\imath=0}^{\infty} e^{-\lambda_{\imath}, p^{t}} \sim(4 \pi t)^{-(n / 2)}\left(a_{0, p}+a_{1, p} t+a_{2, p} t^{2}+\cdots\right), \quad\left(t \rightarrow 0^{+}\right)
$$

here the coefficients $a_{k, p}, k=0,1,2$ were calculated by Patodi in [11] as follows :

$$
a_{0, p}=\left(\begin{array}{c}
n \\
p
\end{array}\right) \operatorname{vol}(M) \text {, }
$$




$$
\begin{aligned}
& a_{1, p}=\left(\frac{1}{6}\left(\begin{array}{c}
n \\
p
\end{array}\right)-\left(\begin{array}{c}
n-2 \\
p-1
\end{array}\right)\right) \int_{M} \rho d v, \\
& a_{2, p}=\int_{M}\left(c_{1}(n, p) \rho^{2}+c_{2}(n, p)|\tilde{R}|^{2}+c_{3}(n, p)|R|^{2}\right) d v .
\end{aligned}
$$

where $d v$ denotes the volume element of $M$ and

$$
\begin{aligned}
& c_{1}(n, p)=\frac{1}{72}\left(\begin{array}{l}
n \\
p
\end{array}\right)-\frac{1}{6}\left(\begin{array}{c}
n-2 \\
p-1
\end{array}\right)+\frac{1}{2}\left(\begin{array}{c}
n-4 \\
p-2
\end{array}\right) ; \\
& c_{2}(n, p)=-\frac{1}{180}\left(\begin{array}{l}
n \\
p
\end{array}\right)+\frac{1}{2}\left(\begin{array}{c}
n-2 \\
p-1
\end{array}\right)-2\left(\begin{array}{c}
n-4 \\
p-2
\end{array}\right) ; \\
& c_{3}(n, p)=\frac{1}{180}\left(\begin{array}{l}
n \\
p
\end{array}\right)-\frac{1}{12}\left(\begin{array}{c}
n-2 \\
p-1
\end{array}\right)+\frac{1}{2}\left(\begin{array}{c}
n-4 \\
p-2
\end{array}\right),
\end{aligned}
$$

here $\left(\begin{array}{l}l \\ q\end{array}\right)$ is understood to be zero when $l<0$ or $q<0$ or $l<q$.

Now, we are going to recall some fundamental results in the theory of minimal hypersurfaces in an Euclidean Sphere.

Theorem A (Chern, Do Carmo and Kobayashi [4] or Lawson [9]). The Clifford tori $M_{m, n-m}=S^{m}(\sqrt{m / n}) \times S^{n-m}(\sqrt{n-m / n}), m=1, \cdots, n-1$ are the only closed minımal hypersurfaces in $S^{n+1}(1)$ satısfying $S=n$.

THEOREM B (Chang [2] or also Cheng and Wan [3]). A closed minimal hypersurface with constant scalar curvature in $S^{4}(1)$ is either an equatorial 3sphere, a Clifford torus, or a Cartan's minimal hypersurface.

\section{§3. Proof of the theorems}

In this section, we turn to prove the theorems.

Proof of Theorem 1. Since $M$ is a minimal hypersurface (i.e., $h=0$ ) in $S^{4}(1)$, thus from $(2.4)-(2.6)$ we have

$$
\begin{aligned}
\rho & =6-S, \\
|R|^{2} & =2 S^{2}-2 \sum \lambda_{i}^{4}-4 S+12, \\
|\widetilde{R}|^{2} & =\sum \lambda_{i}^{4}+12-4 S .
\end{aligned}
$$

Let $M_{0}$ denote either the totally geodesic 3 -sphere, or Clifford torus $S^{1}(\sqrt{1 / 3}) \times$ $S^{2}(\sqrt{2 / 3})$, or Cartan's minimal hypersurface in $S^{4}(1)$. We know that $M_{0}$ has the constant principal curvatures $\lambda_{i}^{0}(1 \leqq i \leqq 3)$. Let $\rho_{0}, \widetilde{R}_{0}, R_{0}$ and $S_{0}$ denote respectively the scalar curvature, Ricci curvature tensor, Curvature tensor and the square of the length of the second fundamental form of $M_{0}$. Then $\rho_{0}=$ $6-S_{0},\left|\tilde{R}_{0}\right|^{2}=\Sigma\left(\lambda_{\imath}^{0}\right)^{4}+12-4 S_{0},|R|^{2}=2 S_{0}^{2}-2 \Sigma\left(\lambda_{\imath}^{0}\right)^{4}-4 S_{0}+12$ and $S_{0}=\Sigma\left(\lambda_{\imath}^{0}\right)^{2}$. Let 
$a_{k, p}$ and $a_{k, p}^{0}$ be the coefficients of the asymptotic expansion of Minakshisundaram-Pleijel corresponding to $M$ and $M_{0}$ respectively. Since $\operatorname{Spec}^{p}(M)=\operatorname{Spec}^{p}\left(M_{0}\right)$ for a given $p(0 \leqq p \leqq 3)$, we have $a_{k, p}=a_{k, p}^{0}$ for $k=0,1,2$ from the asymptotic expansion formula. Thus, by (2.7)-(2.9), we have

$$
\begin{aligned}
& \operatorname{vol}(M)=\operatorname{vol}\left(M_{0}\right), \\
& \int_{M} \rho d v=\int_{M_{0}} \rho_{0} d v_{0}, \\
& \int_{M}\left(c_{1}(3, p) \rho^{2}+c_{2}(3, p)|\widetilde{R}|^{2}+c_{3}(3, p)|R|^{2}\right) d v \\
& \quad=\int_{M_{0}}\left(c_{1}(3, p) \rho_{0}{ }^{2}+c_{2}(3, p)\left|\tilde{R}_{0}\right|^{2}+c_{3}(3, p)\left|R_{0}\right|^{2}\right) d v_{0} .
\end{aligned}
$$

Here we have used $1 / 6\left(\begin{array}{l}3 \\ p\end{array}\right) \neq\left(\begin{array}{c}1 \\ p-1\end{array}\right)$ for any $p=0,1,2,3$ in (3.5). Substituting (3.1)-(3.3) into (3.6) and making use of (3.4), (3.5), we have

$$
\begin{aligned}
& \int_{M}\left(\left(c_{1}(3, p)+2 c_{3}(3, p)\right) S^{2}+\left(c_{2}(3, p)-2 c_{3}(3, p)\right) \sum \lambda_{\imath}^{4}\right) d v \\
& \quad=\int_{M_{0}}\left(\left(c_{1}(3, p)+2 c_{3}(3, p)\right) S_{0}^{2}+\left(c_{2}(3, p)-2 c_{3}(3, p)\right) \Sigma\left(\lambda_{\imath}^{0}\right)^{4}\right) d v_{0} .
\end{aligned}
$$

Since $M$ and $M_{0}$ are 3-dimensional minimal hypersurfaces in $S^{4}(1)$, we have $\sum \lambda_{\imath}^{4}=(1 / 2) S^{2}$ and $\Sigma\left(\lambda_{2}^{0}\right)^{4}=(1 / 2) S_{0}^{2}$. Hence (3.7) becomes

$$
\left(c_{1}(3, p)+\frac{1}{2} c_{2}(3, p)+c_{3}(3, p)\right)\left(\int_{M} S^{2} d v-\int_{M_{0}} S_{0}^{2} d v_{0}\right)=0 .
$$

Because for any $p \in\{0,1,2,3\}, c_{1}(3, p)+(1 / 2) c_{2}(3, p)+c_{3}(3, p) \neq 0$. So (3.8) implies that

$$
\int_{M} S^{2} d v=\int_{M_{0}} S_{0}^{2} d v_{0}=S_{0}^{2} \operatorname{vol}\left(M_{0}\right) .
$$

On the other hand, (3.5) implies that

$$
\int_{M} S d v=S_{0} \operatorname{vol}\left(M_{0}\right)
$$

Thus, by Schwarz inequality, we get

$$
S_{0} \operatorname{vol}\left(M_{0}\right)=\int_{M} S d v \leqq\left(\int_{M} S^{2} d v\right)^{1 / 2}\left(\int_{M} d v\right)^{1 / 2}=S_{0} \operatorname{vol}\left(M_{0}\right) .
$$

Hence

$$
S=S_{0} \text {, }
$$

i.e. $M$ is a minimal hypersurface in $S^{4}(1)$ with constant scalar curvature. From Theorem B, we obtain that $M$ is either the totally geodesic 3-space (when $S=$ 
$S_{0}=0$ ), or Clifford torus (when $S=S_{0}=3$ ), and or Cartan's minimal hypersurface (when $S=S_{0}=6$ ). This proves $M=M_{0}$.

Proof of Theorem 2. Since $M$ is a minimal hypersurface in $S^{n+1}(1)$, we have

$$
\begin{gathered}
\rho=n(n-1)-S, \\
|R|^{2}=2 S^{2}-2 \sum \lambda_{i}^{4}-4 S+2 n(n-1), \\
|\widetilde{R}|^{2}=\sum \lambda_{i}^{4}+n(n-1)^{2}-2(n-1) S .
\end{gathered}
$$

Specially, for $M_{m, n-m}=S^{m}(\sqrt{m / n}) \times S^{n-m}(\sqrt{n-m / n})(1 \leqq m \leqq n-1)$, it is well known that $S_{0}=n$ and $\Sigma\left(\lambda_{\imath}^{0}\right)^{4}=(n-m)^{3}+m^{3} / n(n-m)$, where $\lambda_{\imath}^{0}$ are the principal curvature of $M_{m, n-m}$. Since $\operatorname{Spec}^{p}(M)=\operatorname{Spec}^{p}\left(M_{m, n-m}\right)$ for $p=0,1$. By the same arguments as in the proof of Theorem 1 , we have

$$
\begin{aligned}
& \operatorname{vol}(M)=\operatorname{vol}\left(M_{m, n-m}\right), \\
& \int_{M} \rho d v=\int_{M_{m, n-m}} \rho_{0} d v_{0},
\end{aligned}
$$

and for $p=0,1$,

$$
\begin{aligned}
& \int_{M}\left(c_{1}(n, p) \rho^{2}+c_{2}(n, p)|\tilde{R}|^{2}+c_{3}(n, p)|R|^{2}\right) d v \\
& \quad=\int_{M_{m, n-m}}\left(c_{1}(n, p) \rho_{0}{ }^{2}+c_{2}(n, p)\left|\tilde{R}_{0}\right|^{2}+c_{3}(n, p)\left|R_{0}\right|^{2}\right) d v_{0} .
\end{aligned}
$$

Substituting $\left(3.1^{\prime}\right)-\left(3.3^{\prime}\right)$ and (3.11) (3.12) into (3.13), we get that, for $p=0,1$,

$$
\begin{aligned}
\int_{M}\left[\left(c_{1}(n, p)+2 c_{3}(n, p)\right) S^{2}+\left(c_{2}(n, p)-2 c_{3}(n, p)\right) \sum \lambda_{\imath}^{4}\right] d v \\
=\left(c_{1}(n, p)+2 c_{3}(n, p)\right) n^{2} \operatorname{vol}\left(M_{m, n-m}\right) \\
\quad+\left(c_{2}(n, p)-2 c_{3}(n, p)\right) \frac{(n-m)^{3}+m^{3}}{n(n-m)} \operatorname{vol}\left(M_{m, n-m}\right) .
\end{aligned}
$$

We regard (3.14) as the linear equations $\int_{M} S^{2} d v$ and $\int_{M} \sum \lambda_{i}^{4} d v$. Since

$$
\operatorname{det}\left(\begin{array}{ll}
c_{1}(n, 0)+2 c_{3}(n, 0) & c_{2}(n, 0)-2 c_{3}(n, 0) \\
c_{1}(n, 1)+2 c_{3}(n, 1) & c_{2}(n, 1)-2 c_{3}(n, 1)
\end{array}\right)=\frac{1}{90} \neq 0,
$$

(3.14) has the unique solutions:

$$
\begin{array}{r}
\int_{M} S^{2} d v=n^{2} \operatorname{vol}\left(M_{m, n-m}\right), \\
\int_{M} \sum \lambda_{i}^{4} d v=\frac{(n-m)^{3}+m^{3}}{n(n-m)} \operatorname{vol}\left(M_{m, n-m}\right) .
\end{array}
$$

On the other hand, from (3.12) we have 


$$
\int_{M} S d v=n \operatorname{vol}\left(M_{m, n-m}\right) .
$$

Thus, from Schwarz inequality, the first equation of (3.15) and (3.16) imply that

$$
S=n \text {. }
$$

i.e. $M$ is a closed minimal hypersurface in $S^{n+1}(1)$ satisfying $S=n$. From Theorem A, we obtain that $M$ is one of $\left\{S^{k}(\sqrt{k / n}) \times S^{n-k}(\sqrt{n-k / n})\right\}_{k=1}^{n-1}$. Among those Clifford tori only $M_{m, n-m}=S^{m}(\sqrt{m / n}) \times S^{n-m}(\sqrt{n-m / n})$ satisfying the second equation of (3.15). Therefore $M$ is nothing but $M_{m, n-m}$.

Proof of Theorem 3. Let $M$ be a minimal hypersurface in $S^{2 n+1}(1)(n \geqq 4)$, and $\operatorname{Spec}^{p}(M)=\operatorname{Spec}^{p}\left(M_{n, m}\right)$ for some $p$. For Clifford torus $M_{n, n}=S^{n}(\sqrt{1 / 2}) \times$ $S^{n}(\sqrt{1 / 2}) \rightarrow S^{2 n+1}(1)$, by a direct calculation, we know that the square of the length of the second fundamental form equals to $2 n$ and the principal curvatures $\lambda_{l}^{0}=1$ for $1 \leqq i \leqq n, \lambda_{l}^{0}=-1$ for $n+1 \leqq i \leqq 2 n$, which lead to $\sum_{\imath=1}^{2 n}\left(\lambda_{\imath}^{0}\right)^{4}=2 n$. Therefore, with the same arguments as in the proof of Theorem 2, we have:

$$
\begin{aligned}
\operatorname{vol}(M) & =\operatorname{vol}\left(M_{n, n}\right) \\
\left(\frac{1}{6}\left(\begin{array}{c}
2 n \\
p
\end{array}\right)-\left(\begin{array}{c}
2 n-2 \\
p-1
\end{array}\right)\right) \int_{M} S d v & =\left(\frac{1}{6}\left(\begin{array}{c}
2 n \\
p
\end{array}\right)-\left(\begin{array}{c}
2 n-2 \\
p-1
\end{array}\right)\right) \int_{M_{n, n}} 2 n d v_{0}
\end{aligned}
$$

and

$$
\begin{aligned}
& \int_{M}\left(c_{1}(2 n, p) \rho^{2}+c_{2}(2 n, p)|\widetilde{R}|^{2}+c_{3}(2 n, p)|R|^{2}\right) d v \\
& \quad=\int_{M_{n, n}}\left(c_{1}(2 n, p) \rho_{0}{ }^{2}+c_{2}(2 n, p)\left|\tilde{R}_{0}\right|^{2}+c_{3}(2 n, p)\left|R_{0}\right|^{2}\right) d v_{0} .
\end{aligned}
$$

The crucial point in this case is to show that there is at least a $p=p(n)$ such that

$$
\begin{gathered}
c_{1}(2 n, p)+2 c_{3}(2 n, p)+\frac{1}{2 n}\left(c_{2}(2 n, p)-2 c_{3}(2 n, p)\right)<0, \\
c_{2}(2 n, p)-2 c_{3}(2 n, p) \geqq 0, \\
\frac{1}{6}\left(\begin{array}{c}
2 n \\
p
\end{array}\right) \neq\left(\begin{array}{c}
2 n-2 \\
p-1
\end{array}\right) .
\end{gathered}
$$

If (3.20) holds for some $p$, we get, from (3.18)-(3.20),

$$
\int_{M} S d v=2 n \operatorname{vol}\left(M_{n, n}\right)
$$

and 


$$
\begin{aligned}
\left(c_{1}+2 c_{3}\right) \int_{M}\left(S^{2}-4 n^{2}\right) d v & =-\left(c_{2}-2 c_{3}\right) \int_{M}\left(\sum \lambda_{i}^{4}-2 n\right) d v \\
& \leqq-\left(c_{2}-2 c_{3}\right) \int_{M}\left(\frac{S^{2}}{2 n}-2 n\right) d v .
\end{aligned}
$$

Here we have used the inequality $\sum_{\imath=1}^{2 n} \lambda_{\imath}^{4} \geqq\left(\left(\sum \lambda_{\imath}^{2}\right)^{2} / 2 n\right)=\left(S^{2} / 2 n\right)$ in (3.21). It is easy to see that (3.21) is equivalent to

$$
\int_{M} S^{2} d v \leqq \int_{M} 4 n^{2} d v
$$

from the first equation of (3.20). Making use of (3.22), (3.18') and Schwarz inequality, we obtain $S=2 n$. By Theorem A, we know that $M$ must be one of the Clifford tori $\left\{M_{m, 2 n-m}\right\}_{1 \leqq m \leqq 2 n-1}$. But from (3.21) we also know that $M$ must satisfy $\int_{M} \sum \lambda_{i}^{4} d v=2 n \operatorname{vol}(M)$. Among the Clifford tori $\left\{M_{m, 2 n-m}\right\}$, only $M_{n, n}=S^{n}(\sqrt{1 / 2}) \times S^{n}(\sqrt{1 / 2})$ satisfies the restriction. This proves $M=M_{n, n}$.

It remains to indicate that there exists at least a $p=p(n)$ satisfying (3.20). When $8 \leqq l=2 n \leqq 40$, we put

$$
p=p(n)=\left\{\begin{array}{lll}
2 & \text { when } l=8,10, \\
3 & \text { when } l=12, \\
1 & \text { when } 14 \leqq l \leqq 40 .
\end{array}\right.
$$

Obviously, for a pair $(l, p)$ given in (3.23) the third equation of (3.20) is satisfied. However, it is interesting to note that $c_{1}(l, p)+2 c_{3}(l, p)>0, c_{2}(l, p)-2 c_{3}(l, p)>0$ except for $l=10$. For $l=10$, there is only $p=2$ (or 8) satisfying $c_{1}(10,2)+$ $2 c_{3}(10,2)=-0.0416 \cdots, c_{2}(10,2)-2 c_{3}(10,2)=1.583 \cdots$, which imply that $c_{1}(10,2)+$ $2 c_{3}(10,2)+(1 / 10)\left(c_{2}(10,2)-2 c_{3}(10,2)\right)=0.116 \cdots>0$. Therefore, any $p$ given in (3.23) satisfies (3.20) in this case. When $l=2 n \geqq 40$, from (2.10) we have

$$
\begin{aligned}
& c_{1}+2 c_{3}=\left(\frac{3 l^{2}-3 l-40 p l+40 p^{2}}{120 p(l-p)} \frac{(l-2)(l-3)}{(p-1)(l-p-1)}+\frac{3}{2}\right)\left(\begin{array}{c}
l-4 \\
p-2
\end{array}\right), \\
& c_{2}-2 c_{3}=\left(\frac{-l^{2}+l+40 p l-40 p^{2}}{60 p(l-p)} \frac{(l-2)(l-3)}{(p-1)(l-p-1)}-3\right)\left(\begin{array}{c}
l-4 \\
p-2
\end{array}\right) .
\end{aligned}
$$

Taking

$$
p=\left[\frac{l-\sqrt{(7 / 10) l^{2}+(3 / 10) l}}{2}\right]\left(\text { or }\left[\frac{l+\sqrt{(7 / 10) l^{2}+(3 / 10) l}}{2}\right]+1\right),
$$

where $[x]$ denotes the biggest integer which is not larger than $x$, by a direct calculation, we conclude

$$
c_{1}(l, p)+2 c_{3}(l, p)>0, \quad c_{2}(l, p)-2 c_{3}(l, p)>0, \quad \frac{1}{6}\left(\begin{array}{c}
l \\
p
\end{array}\right) \neq\left(\begin{array}{c}
l-2 \\
p-1
\end{array}\right),
$$

i.e., (3.20) holds for the $p$. The proof of Theorem 3 is completed. 
Remark. We can also give an analogue discussion in complex version.

Acknowledgement. The author would like to express his gratitude to Professors C.H. Gu and H.S. Hu for their encouragement and to Dr. Q.M. Cheng for helpful discussion.

\section{REFERENCES}

[1] M. Berger, P. Gauduchon et E. Mazet, Le Spactre d'une Variété Riemannienne, Lecture Notes in Math., 194, Springer-Verlag, 1971.

[2] S. CHANG, On minimal hypersurfaces with constant scalar curvature in $S^{4}, \mathrm{~J}$. Differential Geom., 37 (1993), 523-534.

[3] Q.M. Cheng AND X.R. WAN, Hypersurfaces of space form $M^{4}(c)$ with constant mean curvature, Report of the First MSJ International Research Institute on Geometry and Global Analysis, ed. by T. Kotake, S. Nishikawa and R. Schoen, Japan, 1993, 437-442,.

[4] S.S. Chern, M. Do Carmo and S. Kobayashi, Minimal submanifolds of a sphere with second fundamental form of constant length, Functional Analysis and Related Fields, ed. by F. Browder, Springer, Berlin, 1970, 59-75.

[5] H. Donnelly, Spectral invariants of the second variation operator, Illinois J. Math., 21 (1977), 185-189.

[6] P.B. Gilkey, Invariance Theory, the Heat Equation, and the Atiyah-Singer Index Theorem, Math. Lecture Ser., 11, Publish, Wilmington, 1984.

[7] T. Hasegawa, Spectral geometry of closed minimal submanifolds in a space form, real and complex, Kodai Math. J., 3 (1980), 224-252.

[8] A. IKEDA, On spherical space forms which are isospectral but not isometric, J. Math. Soc. Japan, 35 (1983), 437-404.

[9] H.B. LAwson, Local rigidity theorem for minimal hypersurfaces, Ann. of Math., 89 (1969), 187-197.

[10] J. Milnor, Eigenvalues of the Laplace operator of certain manifolds, Proc. Nat. Acad. Sci. USA, 51 no. 4 (1964), 542.

[11] U.K. PATODI, Curvature and the fundamental solution of the heat operator, J. Indian Math. Soc., 34 (1970), 269-285.

[12] S. TANNO AND K. MASUDA, A characterization of the product of two 3-sphere by the spectrum, Kodai Math. J., 8 (1985), 420-429.

[13] M. Vigneras, Variétés riemanniennes isospectrales et non isometriques, Ann. of Math., 112 (1980), 21-32.

Institute of Mathematics

FudAN UNIVERSITY

Shanghai 200433

P.R. CHINA 